Baksa V.P., Bandura A.I., Skaskiv O.B.

\title{
ON EXISTENCE OF MAIN POLYNOMIAL FOR ANALYTIC VECTOR-VALUED FUNCTIONS OF BOUNDED L-INDEX IN THE UNIT BALL
}

\begin{abstract}
In this paper, we present necessary and sufficient conditions of boundedness of $\mathbb{L}$-index in joint variables for vector-functions analytic in the unit ball, where $\mathbf{L}=\left(l_{1}, l_{2}\right): \mathbb{B}^{2} \rightarrow \mathbb{R}_{+}^{2}$ is a positive continuous vector-function, $\mathbb{B}^{2}=\left\{z \in \mathbb{C}^{2}:|z|=\sqrt{\left|z_{1}\right|^{2}+\left|z_{2}\right|^{2}} \leq 1\right\}$. These conditions describe local behavior of homogeneous polynomials (so-called a main polynomial) with power series expansion for analytic vector-valued functions in the unit ball. These results use a bidisc exhaustion of a unit ball.

Key words and phrases: bounded index, bounded L-index in joint variables, analytic function, unit ball, main polynomial, homogeneous polynomial.
\end{abstract}

Department of Mechanics and Mathematics, Ivan Franko National University of Lviv, Lviv, Ukraine (Baksa V.P., Skaskiv O.B.)

Department of Advanced Mathematics,

Ivano-Frankivsk National Technical University of Oil and Gas, Ivano-Frankivsk, Ukraine (Bandura A. I.)

e-mail: vitalinabaksa@gmail.com (BaksaV.P.), andriykopanytsia@gmail.com (Bandura A.I.), olskask@gmail.com (Skaskiv O.B.)

\section{INTRODUCTION}

We need some standard notations (for example see $[5,4,6]$ ). Let $\mathbb{R}_{+}=[0 ;+\infty)$, $\mathbf{0}=$ $(0,0) \in \mathbb{R}_{+}^{2}, \mathbf{1}=(1,1) \in \mathbb{R}_{+}^{2}, R=\left(r_{1}, r_{2}\right) \in \mathbb{R}_{+}^{2},|(z, \omega)|=\sqrt{|z|^{2}+|\omega|^{2}}$. For $A=\left(a_{1}, a_{2}\right) \in$ $\mathbb{R}^{2}, B=\left(b_{1}, b_{2}\right) \in \mathbb{R}^{2}$, we will use formal notations without violation of the existence of these expressions: $A B=\left(a_{1} b_{1}, a_{2} b_{2}\right), A / B=\left(a_{1} / b_{1}, a_{2} / b_{2}\right), A^{B}=\left(a_{1}^{b_{1}}, a_{2}^{b_{2}}\right)$, and the notation $A<B$ means that $a_{j}<b_{j}, j \in\{1,2\}$; the relation $A \leq B$ is defined in the similar way. For $K=\left(k_{1}, k_{2}\right) \in \mathbb{Z}_{+}^{2}$ let us denote $K !=k_{1} ! \cdot k_{2}$ !. Addition, multiplication by scalar and conjugation in $\mathbb{C}^{2}$ is defined componentwise. For $z \in \mathbb{C}^{2}, w \in \mathbb{C}^{2}$ we define $\langle z, w\rangle=z_{1} \bar{w}_{1}+z_{2} \bar{w}_{2}$, where $\bar{w}_{1}, \bar{w}_{2}$ is the complex conjugate of $w_{1}, w_{2}$.

The bidisc $\left\{(z, \omega) \in \mathbb{C}^{2}:\left|z-z_{0}\right|<r_{1},\left|\omega-\omega_{0}\right|<r_{2}\right\}$ is denoted by $\mathbb{D}^{2}\left(\left(z_{0}, \omega_{0}\right), R\right)$, its skeleton $\left\{(z, \omega) \in \mathbb{C}^{2}:\left|z-z_{0}\right|=r_{1},\left|\omega-\omega_{0}\right|=r_{2}\right\}$ is denoted by $\mathbb{T}^{2}\left(\left(z_{0}, \omega_{0}\right), R\right)$, the closed polydisc $\left\{(z, \omega) \in \mathbb{C}^{2}:\left|z-z_{0}\right| \leq r_{1},\left|\omega-\omega_{0}\right| \leq r_{2}\right\}$ is denoted by $\mathbb{D}^{2}\left[\left(z_{0}, \omega_{0}\right), R\right], \mathbb{D}^{2}=$

УДК 517.55

2010 Mathematics Subject Classification: 32A10, 32A17, 32A37.

(C) Baksa V.P., Bandura A.I., Skaskiv O.B., 2019 
$\mathbb{D}^{2}(\mathbf{0} ; \mathbf{1}), \mathbb{D}=\{z \in \mathbb{C}:|z|<1\}$. The open ball $\left\{(z, \omega) \in \mathbb{C}^{2}: \sqrt{\left|z-z_{0}\right|^{2}+\left|\omega-\omega_{0}\right|^{2}}<r\right\}$ is denoted by $\mathbb{B}^{2}\left(\left(z_{0}, \omega_{0}\right), r\right)$, the sphere $\left\{(z, \omega) \in \mathbb{C}^{2}: \sqrt{\left|z-z_{0}\right|^{2}+\left|\omega-\omega_{0}\right|^{2}}=r\right\}$ is denoted by $\mathbb{S}^{2}\left(\left(z_{0}, \omega_{0}\right), r\right)$, and the closed ball $\left\{z \in \mathbb{C}^{2}: \sqrt{\left|z-z_{0}\right|^{2}+\left|\omega_{0}-\omega_{0}\right|^{2}} \leq r\right\}$ is denoted by $\mathbb{B}^{2}\left[\left(z_{0}, \omega_{0}\right), r\right], \quad \mathbb{B}^{2}=\mathbb{B}^{2}(\mathbf{0}, \mathbf{1}), \quad \mathbb{D}=\mathbb{B}^{1}=\{z \in \mathbb{C}:|z|<1\}$.

Let $F(z, \omega)=\left(f_{1}(z, \omega), f_{2}(z, \omega)\right)$ be an analytic vector-function in $\mathbb{B}^{2}$. Then at a point $(a, b) \in \mathbb{B}^{2}$ the function $F(z, \omega)$ has a bivariate Taylor expansion:

$$
F(z, \omega)=\sum_{k=0}^{\infty} \sum_{m=0}^{\infty} C_{k l}(z-a)^{k}(\omega-b)^{m},
$$

where $\quad C_{k m}=\left.\frac{1}{k ! m !}\left(\frac{\partial^{k+m} f_{1}(z, \omega)}{\partial z^{k} \partial \omega^{m}}, \frac{\partial^{k+m} f_{2}(z, \omega)}{\partial z^{k} \partial \omega^{m}}\right)\right|_{z=a, \omega=b}=\frac{1}{k ! m !} F^{(k, m)}(a, b)$.

Let $\mathbf{L}(z, \omega)=\left(l_{1}(z, \omega), l_{2}(z, \omega)\right)$, where $l_{j}(z, \omega): \mathbb{B}^{2} \rightarrow \mathbb{R}_{+}^{2}$ is a positive continuous function such that

$$
\forall(z, \omega) \in \mathbb{B}^{2}: \quad l_{j}(z, \omega)>\frac{\beta}{1-\sqrt{|z|^{2}+|\omega|^{2}}},
$$

$j \in\{1,2\}$, where $\beta>\sqrt{2}$ is a some constant.

The norm for the vector-function $F: \mathbb{B}^{2} \rightarrow \mathbb{C}^{2}$ is defined as the sup-norm:

$$
\|F(z, \omega)\|=\max _{1 \leq j \leq 2}\left\{\left|f_{j}(z, \omega)\right|\right\} .
$$

We write

$$
F^{(i, j)}(z, \omega)=\frac{\partial^{i+j} F(z, \omega)}{\partial z^{i} \partial \omega^{j}}=\left(\frac{\partial^{i+j} f_{1}(z, \omega)}{\partial z^{i} \partial \omega^{j}}, \frac{\partial^{i+j} f_{2}(z, \omega)}{\partial z^{i} \partial \omega^{j}}\right) .
$$

An analytic vector-function $F: \mathbb{B}^{2} \rightarrow \mathbb{C}^{2}$ is said to be of bounded $\mathbf{L}$-index (in joint variables) $[1,2,3]$, if there exists $n_{0} \in \mathbb{Z}_{+}$such that

$$
\begin{gathered}
\forall(z, \omega) \in \mathbb{B}^{2} \forall(i, j) \in \mathbb{Z}_{+}^{2}: \\
\frac{\left\|F^{(i, j)}(z, \omega)\right\|}{i ! j ! l_{1}^{i}(z, \omega) l_{2}^{j}(z, \omega)} \leq \max \left\{\frac{\left\|F^{(k, m)}(z, \omega)\right\|}{k ! m ! l_{1}^{k}(z, \omega) l_{2}^{m}(z, \omega)}: k, m \in \mathbb{Z}_{+}, k+m \leq n_{0}\right\} .
\end{gathered}
$$

The least such integer $n_{0}$ is called the $\mathbf{L}$-index in joint variables of the vector-function $F$ and is denoted by $N\left(F, \mathbf{L}, \mathbb{B}^{2}\right)$. The concept of boundedness of $\mathbf{L}$-index in joint variables were considered for other classes of analytic functions. They are differed domains of analyticity: the unit ball $[5,4,12,13]$, the polydisc [7, 11], the Cartesian product of the unit disc and complex plane [8], $n$-dimensional complex space [12, 14]. Vector-valued functions of one and several complex variables having bounded index were considered in $[16,18,15,20,19,17]$.

The function class $Q\left(\mathbb{B}^{2}\right)$ is defined as following: $\forall R \in \mathbb{R}_{+}^{2},|R| \leq \beta, j \in\{1,2\}$ :

$$
0<\lambda_{1, j}(R) \leq \lambda_{2, j}(R)<\infty
$$

where

$$
\begin{aligned}
& \lambda_{1, j}(R)=\inf _{\left(z_{0}, \omega_{0}\right) \in \mathbb{B}^{2}} \inf \left\{\frac{l_{j}(z, \omega)}{l_{j}\left(z_{0}, \omega_{0}\right)}:(z, \omega) \in \mathbb{D}^{2}\left[\left(z_{0}, \omega_{0}\right), R / \mathbf{L}\left(z_{0}, \omega_{0}\right)\right]\right\}, \\
& \lambda_{2, j}(R)=\sup _{\left(z_{0}, \omega_{0}\right) \in \mathbb{B}^{2}} \sup \left\{\frac{l_{j}(z, \omega)}{l_{j}\left(z_{0}, \omega_{0}\right)}:(z, \omega) \in \mathbb{D}^{2}\left[\left(z_{0}, \omega_{0}\right), R / \mathbf{L}\left(z_{0}, \omega_{0}\right)\right]\right\} .
\end{aligned}
$$

We need the following theorem. 
Theorem 1 [3]). Let $\mathbf{L} \in Q\left(\mathbb{B}^{2}\right)$. An analytic vector-function $F: \mathbb{B}^{2} \rightarrow \mathbb{C}^{2}$ has bounded L-index in joint variables if and only if there exist $p \in \mathbb{Z}_{+}$and $c \in \mathbb{R}_{+}$such that for each $(z, \omega) \in \mathbb{B}^{2}$ inequality holds

$$
\max \left\{\frac{\left\|F^{(i, j)}(z, \omega)\right\|}{l_{1}^{i}(z, \omega) l_{2}^{j}(z, \omega)}: i+j=p+1\right\} \leq c \max \left\{\frac{\left\|F^{(k, m)}(z, \omega)\right\|}{l_{1}^{k}(z, \omega) l_{2}^{m}(z, \omega)}: k+m \leq p\right\} .
$$

\section{PROPERTIES OF A POWER SERIES EXPANSION OF ANALYTIC VECTOR-FUNCTIONS IN THE UNIT BALL.}

Let $\left(z_{0}, w_{0}\right) \in \mathbb{B}^{2}$. We expand a vector-function $F: \mathbb{B}^{2} \longrightarrow \mathbb{C}^{2}$ in vector-valued power series

$$
F(z, w)=\sum_{k=0}^{\infty} P_{k}\left(z-z_{0}, w-w_{0}\right)=\sum_{k=0}^{\infty} \sum_{i+j=k} B_{i j}\left(z-z_{0}\right)^{i}\left(w-w_{0}\right)^{j}
$$

where $P_{k}$ is a homogeneous polynomial of degree $k$,

$$
B_{i j}=\frac{F^{(i, j)}\left(z_{0}, w_{0}\right)}{i ! j !}=\left(\frac{f_{1}^{(i, j)}\left(z_{0}, w_{0}\right)}{i ! j !}, \frac{f_{2}^{(i, j)}\left(z_{0}, w_{0}\right)}{i ! j !}\right) .
$$

The polynomial $P_{k_{0}}, k_{0} \in \mathbb{Z}_{+}$, is called a main polynomial in series $(6)$ on $\mathbb{T}^{2}\left(\left(z_{0}, w_{0}\right), R\right)$, if for every $(z, w) \in \mathbb{T}^{2}\left(\left(z_{0}, w_{0}\right), R\right)$ inequality holds

$$
\left\|\sum_{k \neq k_{0}} P_{k}\left(z-z_{0}, w-w_{0}\right)\right\| \leq \frac{1}{2} \max \left\{\left\|B_{i, j}\right\| r_{1}^{i} r_{2}^{j}: i+j=k_{0}\right\} .
$$

The following Theorem 2 and 3 have proofs which are similar to proofs of corresponding theorems in $[9,4,10]$.

Theorem 2. Let $\mathbf{L} \in Q\left(\mathbb{B}^{2}\right)$. If an analytic vector-function $F: \mathbb{B}^{2} \rightarrow \mathbb{C}^{2}$ has bounded $L$-index in joint variables then there exists $p \in \mathbb{Z}_{+}$such that for each $d \in\left(0 ; \frac{\beta}{\sqrt{2}}\right]$ there exists $\eta(d) \in(0 ; d)$ such that for each $\left(z_{0}, w_{0}\right) \in \mathbb{B}^{2}$ and for some $r=r\left(d,\left(z_{0}, w_{0}\right)\right) \in$ $(\eta(d), d)$ and some $\nu_{0}=\nu_{0}\left(d,\left(z_{0}, w_{0}\right)\right) \leq p$ the polynomial $p_{\nu_{0}}$ is main in series (6) on $\mathbb{T}^{2}\left(\left(z_{0}, w_{0}\right), \frac{r \mathbf{1}}{\mathbf{L}\left(z_{0}, w_{0}\right)}\right)$.

Proof. Let $F$ be an analytic vector-function of bounded $\mathbf{L}$-index in joint variables with $N=N\left(F, \mathbf{L}, \mathbb{B}^{2}\right)<+\infty$ and $n_{0}$ be the $\mathbf{L}$-index in joint variables at the point $\left(z_{0}, w_{0}\right) \in \mathbb{B}^{2}$, that is $n_{0}$ the least such that inequality $(2)$ holds in $\left(z_{0}, w_{0}\right)$. Then for every $\left(z_{0}, w_{0}\right) \in \mathbb{B}^{2}$ one has $n_{0} \leq N$.

Define

$$
\begin{gathered}
a_{i, j}^{*}=\frac{\left\|B_{i, j}\right\|}{\mathbf{L}^{i, j}\left(z_{0}, w_{0}\right)}=\frac{\left\|F^{i, j}\left(z_{0}, w_{0}\right)\right\|}{i ! j ! \mathbf{L}^{i, j}\left(z_{0}, w_{0}\right)}, \\
a_{\nu}=\max \left\{a_{i, j}^{*}: i+j=\nu\right\}, c=2\left\{(N+3) ! 3 !+(N+1) C_{N+1}^{N}\right\}=2\left\{(N+3) ! 3 !+(N+1)^{2}\right\} .
\end{gathered}
$$


Let $d \in\left(0 ; \frac{\beta}{\sqrt{2}}\right]$ be an arbitrary number. Put $r_{t}=\frac{d}{(d+1) c^{t}}, \mu_{t}=\max \left\{a_{\nu} r_{t}^{\nu}: \nu \in \mathbb{Z}_{+}\right\}$, $s_{t}=\min \left\{\nu: a_{\nu} r_{t}^{\nu}=\mu_{t}\right\}$ for $t \in \mathbb{Z}_{+}$.

Since $\left(z_{0}, w_{0}\right) \in \mathbb{B}^{2}$ is a fixed point, for every $(k, m) \in \mathbb{Z}_{+}^{2}$ inequality $a_{k, m}^{*} \leq \max \left\{a_{i, j}^{*}:\right.$ $\left.i+j \leq n_{0}\right\}$ holds. Then $a_{\nu} \leq a_{n_{0}}$ for every $\nu \in \mathbb{Z}_{+}$. Then for every $\nu>n_{0}$ with $r_{0}<1$ we have $a_{\nu} r_{0}^{\nu}<a_{n_{0}} r_{0}^{n_{0}}$. Thus, $s_{0} \leq n_{0}$. Since $c r_{t}=r_{t-1}$, we obtain $\nu>s_{t-1}\left(r_{t-1}<1\right)$.

$$
a_{s_{t-1}} r_{t}^{s_{t-1}}=a_{s_{t-1}} r_{t-1}^{s_{t-1}} c^{-s_{t-1}}=a_{\nu} r_{t}^{\nu} c^{\nu-s_{t-1}} \geq c a_{\nu} r_{t}^{\nu}
$$

Hence, $s_{t} \leq s_{t-1}$ for every $t \in \mathbb{N}$. Thus,

$$
\begin{gathered}
\mu_{0}=\max \left\{a_{\nu} r_{0}^{\nu}: \nu \leq n_{0}\right\}, \\
\mu_{t}=\max \left\{a_{\nu} r_{t}^{\nu}: \nu \leq s_{t-1}\right\}, t \in \mathbb{N} .
\end{gathered}
$$

Let us introduce additional notations for $t \in \mathbb{N}$

$$
\begin{aligned}
& \mu_{0}^{*}=\max \left\{a_{\nu} r_{0}^{\nu}: s_{0} \neq \nu \leq n_{0}\right\}, s_{0}^{*}=\min \left\{k: k \neq s_{0}, a_{\nu} r_{0}^{\nu}=\mu_{0}^{*}\right\}, \\
& \mu_{t}^{*}=\max \left\{a_{\nu} r_{t}^{\nu}: s_{t} \neq \nu \leq s_{t-1}\right\}, s_{t}^{*}=\min \left\{k: k \neq s_{t}, a_{\nu} r_{t}^{\nu}=\mu_{t}^{*}\right\} .
\end{aligned}
$$

Now we will prove that there exists $t_{0} \in \mathbb{Z}_{+}$, for which

$$
\frac{\mu_{t_{0}}^{*}}{\mu_{t_{0}}} \leq \frac{1}{c}
$$

On the contrary, suppose that for each $t \in \mathbb{Z}_{+}$the next inequality holds

$$
\frac{\mu_{t_{0}}^{*}}{\mu_{t_{0}}}>\frac{1}{c}
$$

For $s_{t}^{*}<s_{t}$ we have

$$
a_{s_{t}^{*}} r_{t+1}^{s_{t}^{*}}=\frac{a_{s_{t}^{*}} r_{t}^{s_{t}^{*}}}{c^{s_{t}^{*}}}=\frac{\mu_{t}^{*}}{c^{s_{t}^{*}}}>\frac{\mu_{t}}{c^{s_{t}^{*}+1}}=\frac{a_{s_{t}} r_{t}^{s_{t}}}{c^{s_{t}^{*}+1}}=\frac{a_{s_{t}} r_{t+1}^{s_{t}}}{c^{s_{t}^{*}+1}-s_{t}} \geq a_{s_{t}} r_{t+1}^{s_{t}} .
$$

For each $\nu>s_{t}^{*}, \nu \neq s_{t}\left(\right.$ that is $\left.\nu-1 \geq s_{t}^{*}\right)$ we educe

$$
a_{s_{t}^{*}} r_{t+1}^{s_{t}^{*}}=\frac{a_{s_{t}^{*}} r_{t}^{s_{t}^{*}}}{c^{s_{t}^{*}}} \geq \frac{a_{\nu} r_{t}^{\nu}}{c^{s_{t}^{*}}} \geq \frac{a_{\nu} r_{t}^{\nu}}{c^{\nu-1}}=c a_{\nu} r_{t+1}^{\nu}
$$

Thus, $a_{s_{t}^{*}} r_{t+1}^{s_{t}^{*}}>a_{\nu} r_{t+1}^{\nu}$ for all $\nu>s_{t}^{*}$. Then

$$
s_{t+1} \leq s_{t}^{*} \leq s_{t}-1
$$

If $s_{t}<s_{t}^{*} \leq s_{t-1}$ then the equality $s_{t+1}=s_{t}$ can be valid. Indeed, $s_{t+1} \leq s_{t}$. And with $s_{t+1}<s_{t}$ we have $s_{t+1}<s_{t-1}$. It implies (10).

Therefore, from inequalities $s_{t+1}^{*} \leq s_{t}$ and $s_{t}^{*} \neq s_{t+1}$ we have $s_{t+1}^{*}<s_{t+1}$. Hence, instead (10) we have

$$
s_{t+2} \leq s_{t+1}^{*} \leq s_{t+1}-1=s_{t}-1 \text {. }
$$


Then, if for all $t \in \mathbb{Z}_{+}$is true (9), then for each $t \in \mathbb{Z}_{+}$one of two is executed: or $s_{t+2} \leq s_{t+1} \leq s_{t}-1$, or $s_{t+2} \leq s_{t}-1, s_{t+2} \leq s_{t}-1$, since $s_{t+2} \leq s_{t+1}$. Then we have

$$
s_{t} \leq s_{t-2}-1 \leq \ldots \leq s_{t-2[/ 2]}-[t / 2] \leq s_{0}-[t / 2] \leq N-[t / 2] .
$$

In other words, $s_{t}<0$ with $t>2 N+1$. It is a contradiction. Therefore, there exists $t_{0} \leq 2 N+1$, for which (8) is true.

Put $r=r_{t_{0}}, \eta(d)=\frac{d}{(d+1) c^{2(N+1)}}, p=N$ and $\nu_{0}=s_{t_{0}}$. Then for all $(i+j) \neq \nu_{0}=s_{t_{0}}$ on $\mathbb{T}^{2}\left(\left(z_{0}, w_{0}\right), \frac{r \mathbf{e}}{\mathbf{L}\left(z_{0}, w_{0}\right)}\right)$ in view of $(7)$ and (8) we obtain that

$$
\left\|B_{i j}\right\|\left|\left(z-z_{0}\right)^{i}\left(w-w_{0}\right)^{j}\right|=a_{i, j}^{*} r^{i+j} \leq a_{i+j} r^{i+j} \leq \frac{1}{c} a_{s_{t_{0}}} r_{t_{0}}^{s_{t_{0}}}=\frac{1}{c} a_{\nu_{0}} r^{\nu_{0}} .
$$

Thus, for $(z, w) \in \mathbb{T}^{2}\left(\left(z_{0}, w_{0}\right), \frac{r \not}{\mathbf{L}\left(z_{0}, w_{0}\right)}\right)$

$$
\begin{gathered}
\left\|\sum_{i+j \neq \nu_{0}} B_{i, j}\left(z-z_{0}\right)^{i}\left(w-w_{0}\right)^{j}\right\| \leq \sum_{i+j \neq \nu_{0}} a_{i, j}^{*} r^{i+j} \leq \sum_{\nu=0}^{\infty} a_{\nu} C_{\nu+1}^{\nu} r_{\nu}= \\
=\sum_{\nu=0}^{s t_{0}-1} a_{\nu} C_{\nu+1}^{\nu} r_{\nu}+\sum_{\nu=s_{t_{0}-1}+11}^{\infty} a_{\nu} C_{\nu+1}^{\nu} r_{\nu} .
\end{gathered}
$$

We estimate two sums in (11). Then in view of inequality (8) we obtain that $\mu_{t_{0}}^{*} \leq \frac{1}{c} \mu_{t_{0}}$ or $\max \left\{a_{\nu} r_{t_{0}}: \nu \neq s_{t_{0}}, \nu \leq s_{t_{0}-1}\right\} \leq \frac{1}{c} \max \left\{a_{\nu} r_{t_{0}}: \nu \neq s_{t_{0}}, \nu \leq s_{t_{0}-1}\right\}$, we have $a_{\nu} r^{\nu} \leq \frac{1}{c} a_{\nu_{0}} r^{\nu_{0}}$. From (10) we have

$$
\sum_{\substack{\nu=0, \nu \neq s_{t_{0}}}}^{s_{t_{0}-1}} a_{\nu} C_{\nu+1}^{\nu} r_{\nu} \leq \frac{a_{\nu_{0}} r^{\nu_{0}}}{c} \sum_{\nu=0}^{N} C_{\nu+1}^{\nu} \leq \frac{a_{\nu_{0}} r^{\nu_{0}}}{c}(N+1)^{2}
$$

For all $\nu \geq s_{t_{0}-1}+1$ we have $a_{\nu} r_{t_{0}-1}^{\nu} \leq \mu_{t_{0}-1}$. Thus, $a_{\nu} r_{t_{0}}^{\nu}=\frac{a_{\nu} r_{t_{0}-1}^{\nu}}{c^{\nu}} \leq \frac{\mu_{t_{0}-1}}{c^{\nu}}$. From (8) we have

$$
\begin{gathered}
\sum_{\nu=s_{t_{0}-1}+1}^{\infty} a_{\nu} C_{\nu+1}^{\nu} r^{\nu} \mu_{t_{0}-1} C_{\nu+1}^{\nu} \frac{1}{c^{\nu}} \leq\left. a_{s_{t_{0}-1}} r_{t_{0}}^{s_{t_{0}-1}} c^{s_{t_{0}-1}}\left(\sum_{\nu=s_{t_{0}-1}+1}^{\infty} x^{\nu+2}\right)^{\prime \prime}\right|_{x=\frac{1}{c}}= \\
=\left.\frac{a_{\nu_{0}} r^{\nu_{0}}}{c} c^{s_{t_{0}-1}}\left\{\frac{x^{s_{t_{0}-1}+3}}{1-x}\right\}^{\prime \prime}\right|_{x=\frac{1}{c}}=\frac{a_{\nu_{0}} r^{\nu_{0}}}{c} c^{s_{t_{0}-1}} \sum_{j=0}^{2} C_{2}^{j} 2 !\left(s_{t_{0}-1}+3\right) \times \ldots \times \\
\times\left.\left(s_{t_{0}-1}+4-j\right) \cdot \frac{x^{s_{t_{0}-1}+3-j}}{(1-x)^{3-j}}\right|_{x=\frac{1}{c}} \leq \frac{a_{\nu_{0}} r^{\nu_{0}}}{c} c^{s_{t_{0}-1}} 2 !(N+3) ! \frac{(1 / c)^{s_{t_{0}-1}+3-j}}{(1-1 / c)^{3-j}}= \\
=2 !(N+3) ! \frac{a_{\nu_{0}} r^{\nu_{0}}}{c} \sum_{j=0}^{2} \frac{1}{(c-1)^{3-j}} \leq 3 !(N+3) ! \frac{a_{\nu_{0}} r^{\nu_{0}}}{c}
\end{gathered}
$$

for $c \geq 2$. Then from (11)-(13) we obtain

$$
\left\|\sum_{i+j \neq \nu_{0}} B_{i, j}\left(z-z_{0}\right)^{i}\left(w-w_{0}\right)^{j}\right\| \leq \frac{\left((N+1) C_{N+1}^{N}+3 !(N+3) !\right) a_{\nu_{0}} r^{\nu_{0}}}{c} \leq \frac{1}{2} a_{\nu_{0}} r^{\nu_{0}} .
$$

Then the polynomial $p_{\nu_{0}}$ is main in $(6)$ on $\mathbb{T}^{2}\left(\left(z_{0}, w_{0}\right), \frac{r \mathbf{e}}{\mathbf{L}\left(z_{0}, w_{0}\right)}\right)$. 
Theorem 3. Let $\mathbf{L} \in Q\left(\mathbb{B}^{2}\right)$. If there exist $p \in \mathbb{Z}_{+}, d \in(0 ; 1], \eta \in(o ; d)$ such that for each $\left(z_{0}, w_{0}\right) \in \mathbb{B}^{2}$ and for some $R=\left(r_{1}, r_{2}\right)$ with $r_{j}=r_{j}\left(d,\left(z_{0}, w_{0}\right)\right) \in(\eta, d), j \in\{1,2\}$ and for some $\nu_{0}=\nu_{0}\left(d,\left(z_{0}, w_{0}\right)\right) \leq p$ a polynomial $p_{\nu_{0}}$ is main in $(6)$ on $\mathbb{T}^{2}\left(\left(z_{0}, w_{0}\right), \frac{R}{\mathbf{L}\left(z_{0}, w_{0}\right)}\right)$, then an analytic vector-function $F: \mathbb{B}^{2} \rightarrow \mathbb{C}^{2}$ has bounded $L$-index in joint variables.

Proof. Suppose that exist $p \in \mathbb{Z}_{+}, d \leq 1$ and $\eta \in(o ; d)$ such that for each $\left(z_{0}, w_{0}\right) \in \mathbb{B}^{2}$ and for some $R=\left(r_{1}, r_{2}\right)$ with $r_{j}=r_{j}\left(d,\left(z_{0}, w_{0}\right)\right) \in(\eta, d), j \in\{1,2\}$ and for some $\nu_{0}=\nu_{0}\left(d,\left(z_{0}, w_{0}\right)\right) \leq p$ polynomial $p_{\nu_{0}}$ is main in (6) in $\mathbb{T}^{2}\left(\left(z_{0}, w_{0}\right), \frac{R}{\mathbf{L}\left(z_{0}, w_{0}\right)}\right)$. We put $r_{0}=\max _{1 \leq j \leq 2} r_{j}$. Then

$$
\begin{aligned}
\left\|\sum_{i+j \neq \nu_{0}} B_{i, j}\left(z-z_{0}\right)^{i}\left(w-w_{0}\right)^{j}\right\|= & \left\|F(z, w)-\sum_{i+j=\nu_{0}} B_{i, j}\left(z-z_{0}\right)^{i}\left(w-w_{0}\right)^{j}\right\| \leq \\
& \leq \frac{a_{\nu_{0}} r_{0}^{\nu_{0}}}{2} .
\end{aligned}
$$

Hence, in view of Cauchy's integral formula we obtain that

$$
\left\|B_{i, j}\left(z-z_{0}\right)^{i}\left(w-w_{0}\right)^{j}\right\|=a_{i, j}^{*} r_{1}^{i} r_{2}^{j} \leq \frac{a_{\nu_{0}} r_{0}^{\nu_{0}}}{2}, \quad \forall i, j \in \mathbb{Z}_{+}^{2}, \quad i+j \neq \nu_{0},
$$

and for all $i+j=\nu \neq \nu_{0}$ one has

$$
a_{\nu} r_{1}^{i} r_{2}^{j} \leq \frac{a_{\nu_{0}} r_{0}^{\nu_{0}}}{2}
$$

Suppose that $F$ is of unbounded L-index in joint variables. By Theorem 1 for each $p_{1} \in \mathbb{Z}_{+}$ and $c>1 \exists\left(z_{0}, w_{0}\right) \in \mathbb{B}^{2}$ such that

$$
\max \left\{\frac{\left\|F^{(i, j)}\left(z_{0}, w_{0}\right)\right\|}{l_{1}^{i}\left(z_{0}, w_{0}\right) l_{2}^{j}\left(z_{0}, w_{0}\right)}: i+j=p_{1}+1\right\}>c \cdot \max \left\{\frac{\left\|F^{(k, m)}\left(z_{0}, w_{0}\right)\right\|}{l_{1}^{k}\left(z_{0}, w_{0}\right) l_{2}^{m}\left(z_{0}, w_{0}\right)}: k+m \leq p_{1}\right\} .
$$

Put $p_{1}=p$ and $c=\left(\frac{(p+1) !}{\eta^{p+1}}\right)^{2}$. Then for $z_{0}\left(p_{1}, c\right), w_{0}\left(p_{1}, c\right)$ one has

$$
\begin{gathered}
\max \left\{\frac{\left\|F^{(i, j)}\left(z_{0}, w_{0}\right)\right\|}{i ! j ! l_{1}^{i}\left(z_{0}, w_{0}\right) l_{2}^{j}\left(z_{0}, w_{0}\right)}: i+j=p_{1}+1\right\}> \\
>\frac{1}{\eta^{p+1}} \max \left\{\frac{\left\|F^{(k, m)}\left(z_{0}, w_{0}\right)\right\|}{k ! m ! l_{1}^{k}\left(z_{0}, w_{0}\right) l_{2}^{m}\left(z_{0}, w_{0}\right)}: k+m \leq p\right\},
\end{gathered}
$$

that is, $a_{p+1}>\frac{a_{\nu_{0}}}{\eta^{p+1}}$. We obtain $a_{p+1} r_{0}^{p+1}>\frac{a_{\nu_{0}} r_{0}^{p+1}}{\eta^{p+1}} \geq a_{\nu_{0}} r_{\nu_{0}}$.

The last inequality contradicts (14). Thus, the vector-function $F$ has bounded L-index in joint variables.

\section{REFERENCES}

[1] Baksa V.P., Analytic vector-functions in the unit ball having bounded $\mathbf{L}$-index in joint variables, Carpathian Mathematical Publications (in print). 
[2] Baksa V.P., Bandura A.I., Skaskiv O.B., Analogs of Fricke's theorems for analytic vector-valued functions in the unit ball having bounded $\mathbf{L}$-index in joint variables, submitted to Proceedings of IAMM of NASU.

[3] Baksa V.P., Bandura A.I., Skaskiv O.B., Analogs of Hayman's theorem and of logarithmic criterion for analytic vector-valued functions in the unit ball having bounded $\mathbf{L}$-index in joint variables. submitted to Matematica Slovaca.

[4] Bandura A. I., Skaskiv O. B. Analytic functions in the unit ball of bounded L-index: asymptotic and local properties. Mat. Stud. 2017, 48 (1), 37-73. doi: 10.15330/ms.48.1.37-73

[5] Bandura A., Skaskiv O. Sufficient conditions of boundedness of L-index and analog of Hayman's Theorem for analytic functions in a ball. Stud. Univ. Babeş-Bolyai Math. 2018, 63 (4), $483-501$. doi:10.24193/subbmath.2018.4.06

[6] Bandura A., Skaskiv O. Functions analytic in the unit ball having bounded L-index in a direction. Rocky Mountain J. Math. 2019, 49 (4), 1063-1092. doi: 10.1216/RMJ-2019-49-4-1063

[7] Bandura A., Petrechko N., Skaskiv O. Maximum modulus in a bidisc of analytic functions of bounded L-index and an analogue of Hayman's theorem. Mat. Bohemica 2018, 143 (4), 339-354. doi: 10.21136/MB.2017.0110-16

[8] Bandura A.I., Skaskiv O.B., Tsvigun V.L. Some characteristic properties of analytic functions in $\mathbb{D} \times \mathbb{C}$ of bounded L-index in joint variables. Bukovyn. Mat. Zh. 2018, 6 (1-2), 21-31.

[9] Bandura A., Petrechko N. Properties of power series expansion of entire function of bounded $\mathbf{L}$-index in joint variables. Visn. Lviv. Un-ty. Ser. Mech.-Math. 2016, Iss. 82, 27-33.

[10] Bandura A.I., Petrechko N.V. Properties of power series of analytic in a bidisc functions of bounded L-index in joint variables. Carpathian Math. Publ. 2017, 9 (1), 6-12. doi: 10.15330/cmp.9.1.6-12

[11] Bandura A.I., Petrechko N.V., Skaskiv O.B. Analytic in a polydisc functions of bounded L-index in joint variables. Mat. Stud. 2016, 46 (1), 72-80. doi: 10.15330/ms.46.1.72-80

[12] Bandura A., Skaskiv O. Analytic functions in the unit ball of bounded L-index in joint variables and of bounded L-index in direction: a connection between these classes. Demonstr. Math. 2019, 52 (1), 82-87. doi: 10.1515/dema-2019-0008

[13] Bandura A.I., Skaskiv O.B. Partial logarithmic derivatives and distribution of zeros of analytic functions in the unit ball of bounded L-index in joint variables. J. Math. Sci. 2019, 239 (1), 17-29. doi: $10.1007 / \mathrm{s} 10958-019-04284-\mathrm{z}$

[14] Bandura A.I., Skaskiv O.B. Exhaustion by balls and entire functions of bounded L-index in joint variables, Ufa Math. J. 2019, 11 (1), 100-113. doi: 10.13108/2019-11-1-100

[15] Bordulyak M.T., Sheremeta M.M. Boundedness of l-index of analytic curves. Mat. Stud. 2011, 36 (2), 152-161.

[16] Heath L. F. Vector-valued entire functions of bounded index satisfying a differential equation. Journal of Research of NBS 1978, 83B (1), 75-79.

[17] Nuray F., Patterson R.F. Vector-valued bivariate entire functions of bounded index satisfying a system of differential equations. Mat. Stud. 2018, 49 (1), 67-74. doi: 10.15330/ms.49.1.67-74

[18] Roy R., Shah S.M. Growth properties of vector entire functions satisfying differential equations. Indian J. Math. 1986, 28 (1), 25-35.

[19] Roy R., Shah S.M. Vector-valued entire functions satisfying a differential equation. J. Math. Anal. Appl. 1986, 116 (2), 349-362.

[20] Sheremeta M. Boundedness of $l-M$-index of analytic curves. Visnyk Lviv Un-ty. Ser. Mech.-Math. Iss. 75, 226-231 (2011) 
Бакса В.П., Бандура А.І., Скасків О.Б. Про існування головного багаточлена для аналітичних в одиничній кулі векторнозначних функиій обмеженого $\mathbf{L}$-індексу за сукупністю змінних // Буковинський матем. журнал - 2019. - Т.7, №2. - С. 6-13.

$\mathrm{У}$ цій статті отримано необхідні і достатні умови обмеженості $\mathbb{L}$-індексу за сукупністю змінних для векторнозначних функій, аналітичних в одиничній кулі, де $\mathbf{L}=\left(l_{1}, l_{2}\right): \mathbb{B}^{2} \rightarrow$ $\mathbb{R}_{+}^{2}$ - додатна неперервна вектор-функція, $\mathbb{B}^{2}=\left\{z \in \mathbb{C}^{2}:|z|=\sqrt{\left|z_{1}\right|^{2}+\left|z_{2}\right|^{2}} \leq 1\right\}$. Ці умови описують локальне поводження однорідних багаточленів (так званих головних багаточленів) з розвинення у степеневий ряд аналітичних в одиничній кулі векторнозначних функцій. Отримані результати використовують бікругове вичерпання одиничної кулі. 